\title{
RECENT BABAR RESULTS ON B DECAYS
}

\author{
P. J. CLARK \\ (for the BABAR Collaboration) \\ School of Physics, University of Edinburgh, Edinburgh, EH9 3JZ, UK \\ P.J.Clark@ed.ac.uk
}

\begin{abstract}
Several recent key results from the BABAR experiment are presented, most using $383.6 \mathrm{fb}^{-1}$ of data. In particular, the search for $B^{+} \rightarrow \tau^{+} \nu$, inclusive and exclusive measurements of $\left|V_{u b}\right|$, measurements of $b \rightarrow d \gamma$ decays and new observations of rare charmless hadronic decays. The new results provide important experimental constraints on the Standard Model and new physics models.
\end{abstract}

Keywords: B decays; flavor; leptonic; semi-leptonic, radiative, hadronic.

PACS numbers: 13.20.He, 14.40.Nd, 11.30.Hv, 13.20.-V, 13.30.CE, 13.30.Eg

\section{Introduction}

The large number of different $B$ meson decays provides a rich environment for studying flavor physics and testing the Standard Model (SM). Several of the processes described in this paper provide interesting constraints on new physics which may be discovered at the Large Hadron Collider (LHC). Since there are numerous $B$ decay measurements, we have focused on recent key results from four subcategories of $B$ decay: purely leptonic $\left(B^{+} \rightarrow \tau^{+} \nu\right)$, semi-leptonic $\left(\left|V_{u b}\right|\right)$, radiative $(b \rightarrow d \gamma)$ and several rare charmless hadronic decays. Charge conjugate processes are assumed throughout. Most of the results presented are obtained from a dataset of $383.6 \pm 4.2$ million $\Upsilon(4 S) \rightarrow B \bar{B}$ events corresponding to an integrated luminosity of $346 \mathrm{fb}^{-1}$. The data are collected by the BABAR detector ${ }^{1}$ at the PEP-II $e^{+} e^{-}$asymmetric $B$ Factory tuned to the center-of-mass energy $\sqrt{s}=10.8 \mathrm{GeV}$. An additional $37 \mathrm{fb}^{-1}$ of data is taken $40 \mathrm{MeV}$ below the $\Upsilon(4 S)$ resonance ("offresonance") for background studies. All unpublished results should be considered preliminary.

\section{2. $B^{+} \rightarrow \tau^{+} \nu$}

The purely leptonic decay, $B^{+} \rightarrow \tau^{+} \nu$, in the SM occurs from quark annihilation via a $W^{+}$boson. The predicted branching fraction is given by: ${ }^{2}$

$$
\mathcal{B}_{S M}\left(B^{+} \rightarrow \tau^{+} \nu\right)=\frac{G_{F}^{2} m_{B^{+}} m_{\tau^{+}}^{2}}{8 \pi}\left[1-\frac{m_{\tau^{+}}^{2}}{m_{B^{+}}^{2}}\right]^{2}\left(f_{B}^{2}\left|V_{u b}\right|^{2} \tau_{B^{+}}\right)
$$


where $G_{F}$ is the Fermi constant, $m_{B}$ and $m_{\tau}$ are the $B^{+}$and $\tau$ meson masses, $f_{B}$ is the $B$ meson decay constant and $\tau_{B}^{+}$is the $B^{+}$lifetime.

The $B^{+} \rightarrow \tau^{+} \nu$ decay is challenging because the final state contains two or more neutrinos. It lacks the usual signal $B$ mass and beam energy constraints to reduce continuum and mis-reconstructed $B$ background. To combat this we use the "recoil" method. The $\Upsilon(4 S)$ resonance produces two B mesons and we reconstruct fully or partially the other $B$ meson, called the "tag" $B$. This is done using the common decays $B^{-} \rightarrow D^{0} l^{-} \nu X^{0}$ (semi-leptonic tag) and $B^{-} \rightarrow D^{0(*)} X^{-}$(hadronic tag). The remaining particles are attributed to the signal $B$ meson. Results for the semileptonic $^{6}$ and hadronic ${ }^{7}$ tag approaches from BABAR are shown in Table 1.

Table 1. $B^{+} \rightarrow \tau^{+} \nu$ branching fractions.

\begin{tabular}{lccc}
\hline$B^{+} \rightarrow \tau^{+} \nu$ & Branching fraction $\left(10^{-4}\right)$ & Significance & $\left(f_{B}\left|V_{u b}\right|\right) 10^{-4}$ \\
\hline Semi-leptonic & $0.9 \pm 0.6 \pm 0.1$ & 1.3 & $7.2_{-2.8}^{+2.0} \pm 0.2$ \\
Hadronic & $1.8_{-0.8}^{+0.9} \pm 0.4 \pm 0.2$ & 2.2 & $10.1_{-2.5-1.5}^{+2.3+1.2}$ \\
Combined & $1.2 \pm 0.4 \pm 0.3 \pm 0.2$ & 2.6 & - \\
\hline
\end{tabular}

These results are consistent with the first evidence seen by the Belle and provide constraints on the $\mathrm{SM}^{19}$ (see Fig. 1 ). $B^{+} \rightarrow \tau^{+} \nu$ is sensitive to possible intermediate charged Higgs bosons ${ }^{2-4}$. Exclusion regions (95\% CL) for this new physics scenario are shown in Fig. 1.
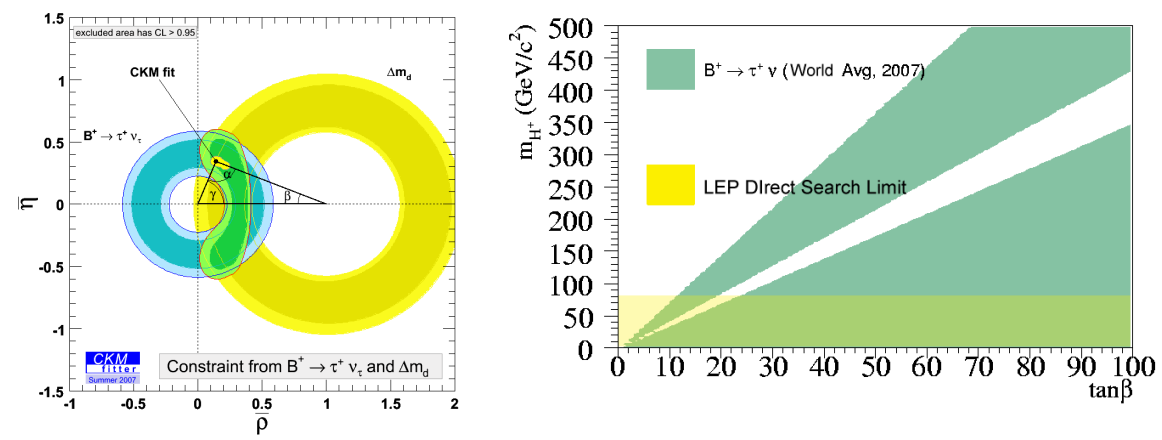

Fig. 1. Constraints from $B^{+} \rightarrow \tau^{+} \nu$ on the Standard Model $\rho-\eta$ plane (left) and exclusion regions for $\tan \beta$ vs charged Higgs mass from $B^{+} \rightarrow \tau^{+} \nu$ (right).

\section{Measurement of $\left|V_{u b}\right|$}

A key goal of the $B$ factories is to over-constrain the SM flavour sector. The dominant constraint comes from $\sin 2 \beta$. To over-constrain, and thus test the $\mathrm{SM},\left|V_{u b}\right|$ should be measured as precisely as possible and compared to that from the SM fit. 
Measuring $\left|V_{u b}\right|$ is experimentally challenging, due to the neutrino presence and the large $b \rightarrow c \ell \nu$ background. There are inclusive and exclusive approaches. For both we can use the recoil method, mentioned earlier, called the "tagged" result.

Exclusively, $\left|V_{u b}\right|$ is obtained from the $B \rightarrow \pi \ell \nu$ branching fraction. The final state has good purity, but suffers from statistical errors due to the smaller branching fraction and theoretical errors to take into account the hadronic uncertainties in the form factor ${ }^{8}$. In the tagged measurement three bins in $q^{2}$ are taken (where $q^{2}$ is the squared invariant mass of the lepton-neutrino system). In the untagged measurement 12 bins are used. The results are shown in Table 2 for $211 \mathrm{fb}^{-1} 9,10$.

Table 2. $\quad B \rightarrow \pi \ell \nu$ branching fractions and $\left|V_{u b}\right|$ (third error due to form factor).

\begin{tabular}{lcc}
\hline$B^{0} \rightarrow \pi^{-} \ell^{+} \bar{\nu}$ & Branching fraction $\left(10^{-4}\right)$ & $\left|V_{u b}\right|\left(10^{-3}\right)$ \\
\hline untagged & $1.46 \pm 0.07 \pm 0.08$ & $3.45_{-0.18-0.14-0.39}^{+0.17+0.13+0.60}$ \\
tagged & $1.33 \pm 0.17 \pm 0.11$ & $3.8_{-0.4-0.3-0.4}^{+0.4+0.2+0.7}$ \\
\hline
\end{tabular}

For the inclusive measurement strong kinematic cuts are needed and the full rate isn't measured. Theoretical calculation of the differential width is required extract $\left|V_{u b}\right|$. Significant progress has been made on this front over the past few years ${ }^{11,12}$. The various kinematic $B A B A R\left|V_{u b}\right|$ measurements ${ }^{13}$ (using a tagged approach) help lead to the world average for inclusive $\left.\left|V_{u b}\right|=4.32 \pm 0.17 \pm 0.35\right) \times 10^{-3}$, for more details see Ref. 14. The world inclusive measurement shows a small discrepancy from the exclusive measurement, and the global SM fit ${ }^{18}$, see Fig. 2.

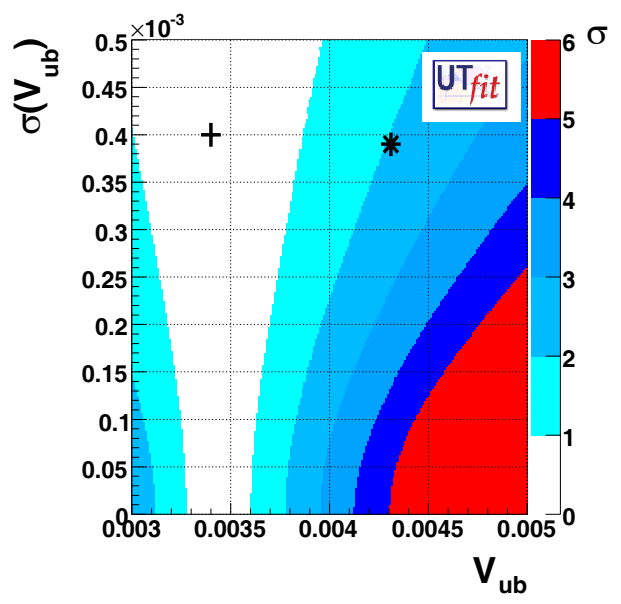

Fig. 2. $\left|V_{u b}\right|$ from: SM fit (contours), exclusive $(+)$ and inclusive $\left({ }^{*}\right)$ measurements. 


\section{4. $b \rightarrow d \gamma$}

The decay $b \rightarrow d \gamma$, being a Flavor Changing Neutral Current (FCNC), is sensitive to new physics beyond the SM. However, it is rare, suppressed by $\left(\left|V_{t d}\right| /\left|V_{t s}\right|\right)^{2}$ relative to $b \rightarrow s \gamma$ decays. The exclusive decay $B \rightarrow(\rho, \omega) \gamma$ was first observed by Belle $^{15}$ and recent $B A B A R$ results $^{16}$ are given in Table 3.

Table 3. Branching fraction results for $b \rightarrow d \gamma$ from $316 \mathrm{fb}^{-1}$ (final row is from an isospin averaged fit).

\begin{tabular}{lccc}
\hline Mode & $N_{\text {sig }}$ & Significance $(\sigma)$ & $\mathcal{B}\left(10^{-6}\right)$ \\
\hline$B^{+} \rightarrow \rho^{+} \gamma$ & $42.0_{-12.7}^{+14.0}$ & 3.8 & $1.10_{-0.33}^{+0.37} \pm 0.09$ \\
$B^{0} \rightarrow \rho^{0} \gamma$ & $38.7_{-9.8}^{+10.6}$ & 4.9 & $0.79_{-0.20}^{+0.22} \pm 0.06$ \\
$B^{0} \rightarrow \omega \gamma$ & $11.0_{-5.6}^{+6.7}$ & 2.2 & $0.40_{-0.20}^{+0.24} \pm 0.05$ \\
$B \rightarrow(\rho, \omega) \gamma$ & - & 6.4 & $1.25_{-0.24}^{+0.25} \pm 0.09$ \\
\hline
\end{tabular}

We can now determine $\left|V_{t d}\right| /\left|V_{t s}\right|$ and compare to that from $\operatorname{CDF}\left(\Delta m_{s}\right)^{17}$ :

$$
\begin{aligned}
& \left|V_{t d}\right| /\left|V_{t s}\right|=0.200_{-0.020}^{+0.021} \pm 0.015 \quad[B A B A R] \\
& \left|V_{t d}\right| /\left|V_{t s}\right|=0.2060 \pm 0.0007_{-0.0060}^{+0.0001} \quad[\mathrm{CDF}]
\end{aligned}
$$

These ratios are in good agreement. Separate charged and neutral fits to the SM $\rho-\eta$ plane $^{18}$ using world averages are shown in Fig. 3.
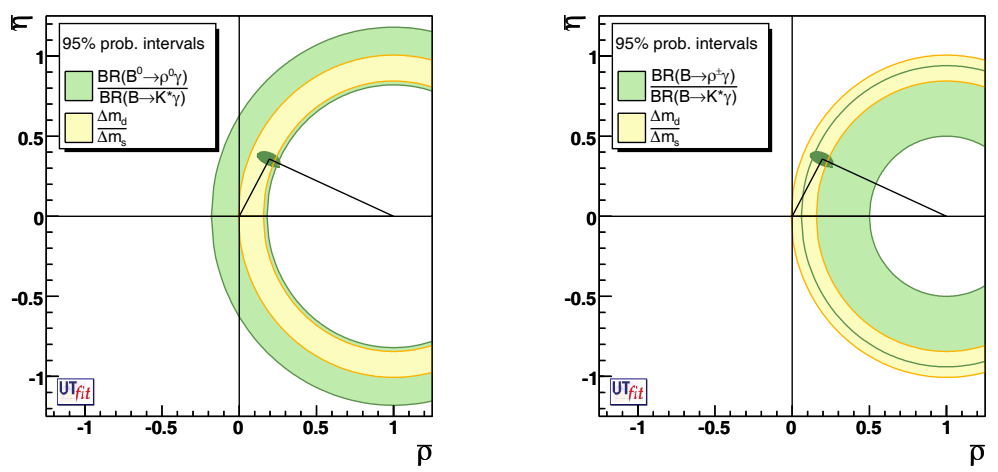

Fig. 3. Bound on the $\rho-\eta$ plane from $\rho \gamma / K^{*} \gamma$ and $\Delta m_{d} / \Delta m_{s}$ measurements.

$B A B A R$ has also searched inclusively for $b \rightarrow d \gamma$ in the kinematic region $1.0<m\left(X_{d}\right)<1.8 \mathrm{GeV} / c^{2}$ using a sum of seven exclusive modes (various $\pi$ and $\eta$ combinations). Using $348 \mathrm{fb}^{-1}$ of data, $178 \pm 53$ signal events were found, representing preliminary first evidence for $b \rightarrow d \gamma$ outside of $\rho$ and $\omega$ resonances:

$$
\left.\sum_{X_{d}=1}^{7} \mathcal{B}\left(B \rightarrow X_{d} \gamma\right)\right|_{1.0<X_{d}<1.8 \mathrm{GeV} / c^{2}}=\left(3.1 \pm 0.9_{-0.5}^{+0.6} \pm 0.5\right) \times 10^{-6} .
$$




\section{Rare Charmless Hadronic Decays}

The $B A B A R$ experiment has published a number rare $B$ decays to charmless hadronic final states ${ }^{14}$. One recent highlight is first observation of $B^{+} \rightarrow K^{+} K^{-} \pi^{+}$. The resonant decays $B^{+} \rightarrow K^{* 0} K^{+}$or $\phi \pi^{+}$are heavily suppressed $b \rightarrow d$ penguins. Previous searches have produced upper limits consistent with no signal. However, using the full $K^{+} K^{-} \pi^{+}$Dalitz region and $347.5 \mathrm{fb}^{-1}$ of data, a substantial signal was observed (429 events). The branching fraction results are given in Table 4 . The $K^{+} K^{-}$invariant mass (see Fig. 4) shows a large broad structure at approximately $1.5 \mathrm{GeV}$. There is also an excess in events at low $K^{-} \pi^{+}$invariant mass. These effects have been seen in other recent mass spectra ${ }^{20-22}$.
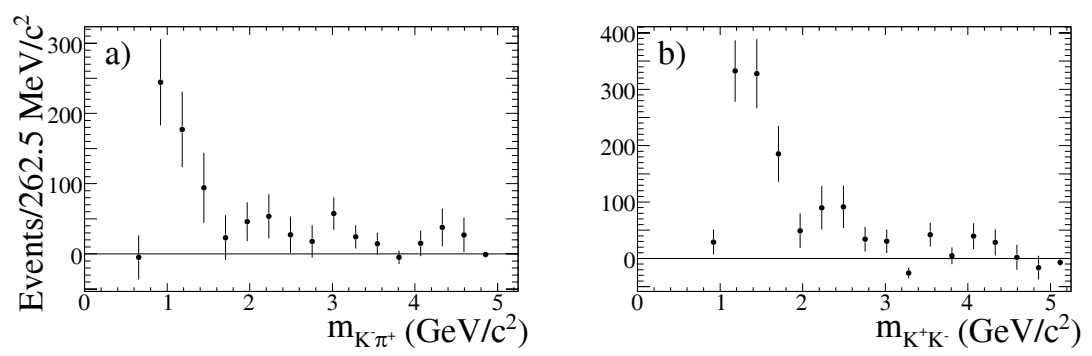

Fig. 4. Invariant mass distributions for $B^{+} \rightarrow K^{+} K^{-} \pi^{+}$.

Some other recent measurements from $B A B A R$ are $B^{0} \rightarrow K^{* 0} \bar{K}^{* 0} 23$ and four $B^{0} \rightarrow K^{* 0} h^{+} h^{-}$decays $^{24}$. Another recent development was the measurement of several axial vector $b_{1}^{0}$ decay modes ${ }^{25}$. All of the results are summarised in Table 4. The $b_{1}^{0} h^{ \pm}$modes are in good agreement with some recent theoretical predictions ${ }^{26}$.

Table 4. Branching fraction results for $b \rightarrow d \gamma$ from $316 \mathrm{fb}^{-1}$ (final row is from an isospin averaged fit).

\begin{tabular}{llr}
\hline Mode & $\mathcal{B}\left(10^{-6}\right)$ & Significance $(\sigma)$ \\
\hline$B^{+} \rightarrow K^{+} K^{-} \pi^{+}$ & $5.0 \pm 0.5 \pm 0.5$ & 9.6 \\
$B^{0} \rightarrow K^{* 0} \bar{K}^{* 0}$ & $1.28_{-0.30}^{+0.35} \pm 0.11$ & 6 \\
$B^{0} \rightarrow K^{* 0} K^{+} K^{-}$ & $27.5 \pm 1.3 \pm 2.2$ & $>10$ \\
$B^{0} \rightarrow K^{* 0} \pi^{+} K^{-}$ & $4.6 \pm 1.1 \pm 0.8$ & 5.3 \\
$B^{0} \rightarrow K^{* 0} K^{+} \pi^{-}$ & $<2.2(90 \% \mathrm{CL})$ & 0.9 \\
$B^{0} \rightarrow K^{* 0} \pi^{+} \pi^{-}$ & $54.5 \pm 2.9 \pm 4.3$ & 10 \\
$B^{+} \rightarrow b_{1}^{0}(1235) \pi^{+}$ & $6.7 \pm 1.7 \pm 1.0$ & 4.0 \\
$B^{+} \rightarrow b_{1}^{0}(1235) K^{+}$ & $9.1 \pm 1.7 \pm 1.0$ & 5.3 \\
$B^{0} \rightarrow b_{1}^{\mp}(1235) \pi^{ \pm}$ & $10.9 \pm 1.2 \pm 0.9$ & 8.9 \\
$B^{0} \rightarrow b_{1}^{-}(1235) K^{+}$ & $7.4 \pm 1.0 \pm 1.0$ & 6.1 \\
\hline
\end{tabular}




\section{Conclusions}

We have presented here a snapshot of recent results from the BABAR experiment. The decay $B^{+} \rightarrow \tau^{+} \nu$ is starting to emerge with the power to constrain two-higgs doublet physics models. A new inclusive $\left|V_{u b}\right|$ measurement using tagged events has improved the world average which now shows a small discrepancy with the global SM fit (still not significant). Using $b \rightarrow d \gamma$ events we have shown interesting comparisons between $\left|V_{t d}\right| /\left|V_{t s}\right|$ obtained from penguin diagrams to that from box diagrams $\left(\Delta m_{S}\right)$. Lastly, we have presented a number of the recently published rare charmless hadronic decays. The observation of a new broad structure at $1.5 \mathrm{GeV} / c^{2}$ in $K^{+} K^{-}$invariant mass is proving interesting. In summary, BABAR is providing precise constraints on the SM and the flavour sector of possible new physics scenarios. Additional data from new-generation flavor experiments and theoretical progress will help continue to this and allow us to probe further.

\section{References}

1. BABAR Collab. (B. Aubert et al.), Nucl. Instrum. Meth. A 479, 1 (2002)

2. W. S. Hou, Phys. Rev. D 48, 2342 (1993)

3. G. Isidori and P. Paradisi, Phys. Lett. B 639, 499 (2006)

4. A. G. Akeroyd and C. H. Chen, Phys. Rev. D 75, 075004 (2007)

5. K. Ikado et al., Phys. Rev. Lett. 97, 251802 (2006)

6. BABAR Collab. (B. Aubert et al.), Phys. Rev. D 76, 052002 (2007)

7. BABAR Collab. (B. Aubert et al.), arXiv:0708.2260 [hep-ex]

8. E. Dalgic et al., Phys. Rev. D 73, 074502 (2006)

9. BABAR Collab. (B. Aubert et al.), Phys. Rev. Lett. 98, 091801 (2006)

10. BABAR Collab. (B. Aubert et al.), Phys. Rev. Lett. 97, 211801 (2006)

11. B.O. Lange, M. Neubert and G. Paz, Phys. Rev. D 72, 073006 (2005)

12. J.R. Andersen and E. Gardi, JHEP 0601, 097 (2006)

13. BABAR Collab. (B. Aubert et al.), arXiv:0708.3702 [hep-ex]

14. Heavy Flavor Averaging Group http://www.slac.stanford.edu/xorg/hfag

15. Belle Collab. (D.Mohapatra et al.) Phys. Rev. Lett. 96, 221601 (2006)

16. BABAR Collab. (B. Aubert et al.), Phys. Rev. Lett. 98, 151802 (2007)

17. CDF Collab. (A. Abulencia et al.) Phys. Rev. Lett. 97, 242003 (2006)

18. M. Bona et al. (UTfit collab.) http://www.utfit.org/

19. J. Charles et al., (CKMfitter Group) Eur. Phys. J. C 41, 1 (2005)

20. Belle Collab (A. Garmash et al.) Phys. Rev. D 71, 092003 (2005)

21. BABAR Collab. (B. Aubert et al.), Phys. Rev. D 74, 032003 (2006)

22. BABAR Collab. (B. Aubert et al.), Phys. Rev. Lett. 99, 161802 (2007)

23. BABAR Collab. (B. Aubert et al.) arXiv:0708.2248 [hep-ex]

24. BABAR Collab. (B. Aubert et al.) Phys. Rev. D 76, 071104 (2007)

25. BABAR Collab. (B. Aubert et al.) Phys. Rev. Lett. 99, 241803 (2007)

26. H. Y. Cheng and K. C. Yang, Phys. Rev. D 76, 114020 (2007) 